\title{
Desfechos das técnicas de Iso Stretching e Pompage em pacientes com doença pulmonar obstrutiva crônica
}

\author{
Adriane Schmidt Pasqualoto* Darlene Costa de Bittencourt*

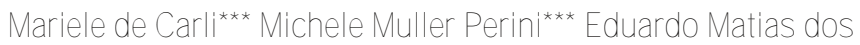 \\ Santos Steidl**** Vívian da Pieve Antunes****
}

Resumo: Objetivo - 0 objetivo do presente estudo foi avaliar os desfechos do uso associado das técnicas de Iso Stretching e pompage sobre a capacidade funcional, força muscular respiratória e a mobilidade torácica de indivíduos portadores de doença pulmonar obstrutiva crônica. Métodos - Tratase de um estudo piloto, onde participaram 10 indivíduos, randomizados em grupo tratado e grupo controle. Foram analisadas as variáveis espirométricas, antropométricas, força muscular respiratória e a capacidade funcional. Da amostra, cinco pacientes concluíram o tratamento de quatro semanas de intervenção com as técnicas de Iso Stretching e pompage. Resultados - Após a intervenção, houve aumento na força muscular inspiratória e expiratória para todos os indivíduos do grupo tratado, bem como, aumento na distância percorrida no TC6 e na expansibilidade torácica. Conclusão - um programa terapêutico baseado nas técnicas de Iso Stretching e pompage por quatro semanas, evidenciaram principalmente 0 aumento na força muscular respiratória, expansibilidade e distância percorrida no TC6.

Descritores: Reabilitação, Modalidades de Fisioterapia, DPOC.

\section{Outcomes of Iso Stretching and Pompage techniques in patients with chronic obstructive pulmonary disease}

\begin{abstract}
Objective - The aim of this study was to evaluate the outcomes of the use of techniques associated Iso Stretching and pompage on functional capacity, respiratory muscle strength and thoracic mobility of individuals with COPD. Methods - This is a pilot study, which involved 10 subjects randomized into treatment group and control group. Were analyzed spirometric, anthropometric, respiratory muscle strength and functional capacity. Sample, five patients completed the four-week treatment intervention with techniques Iso Stretching and pompage. Results - After the intervention, there was an increase in inspiratory and expiratory muscle strength for all individuals in the treated group, as well as increase in 6MWD and chest expansion. Conclusion - a therapeutic program based on the techniques of pompage and Iso Stretching for four weeks, showed mainly the increase in respiratory muscle strength, expandability and distance walked during the 6MWT.
\end{abstract}

Descriptors: Rehabilitation, Physical Therapy Modalities, COPD.

*Doutora em Ciências Pneumológicas pela Universidade Federal do Rio Grande do Sul (UFRGS), Porto Alegre, RS, Brasil.

**Mestre em Ciências Pneumológicas pela Universidade Federal do Rio Grande do Sul (UFRGS), Porto Alegre, RS, Brasil.

***Fisioterapeuta pela Universidade do Universidade Regional do Noroeste do Estado do Rio Grande do Sul (UNIJUÍ), ljuí, RS, Brasil.

****Mestrando em Distúrbios da Comunicação Humana na Universidade Federal de Santa Maria (UFSM), Santa Maria, RS, Brasil.

Rev. Saúde (Santa Maria), Santa Maria, v39, n.2, p.161-170 Jul./Dez.2013. 


\section{Introdução}

A doença pulmonar obstrutiva crônica (DPOC) é uma doença progressiva que se caracteriza pela limitação do fluxo aéreo e por uma resposta inflamatória sistêmica que culmina em importantes manifestações extrapulmonares ${ }^{1}$. Dentre estas manifestações é possivel destacar as alterações musculares esqueléticas, que repercutem sobre a limitação nas atividades de vida diária com consequente descondicionamento muscular periférico.

Cronicamente, o processo fisiopatológico da doença tende a levar à hiperinsuflação pulmonar, que inicialmente reduzirá a capacidade física aos grandes esforços e, posteriormente, ao repouso. Decorrente da hiperinsuflação pulmonar ocorre adaptações estruturais nos músculos da caixa torácica, especialmente do diafragma, que tende a se retificar, diminuindo a zona de aposição e, consequentemente, restrição em sua excursão². Adicionalmente, ocorre a perda do recolhimento elástico, restrição da mobilidade da caixa torácica, recrutamento dos músculos acessórios da inspiração, graus variados de fraqueza muscular periférica e diminuição da resistência muscular, contribuindo para a limitação funcional desses pacientes $3,4,5$.

Para o acompanhamento desses indivíduos a reabilitação pulmonar tem sido a principal recomendação na terapêutica com evidências sólidas na literatura. Autores têm ressaltado a contribuição do treinamento muscular dos membros inferiores, superiores, bem como, treinamento muscular respiratório aos portadores de DPOC e outras pneumopatias crônicas. Entretanto as técnicas de terapia manual, como pompage e, as posturais, como o Iso Stretching, tem sido pouco investigadas como possibilidade terapêutica nessa população ${ }^{6}$.

Embora haja uma série de técnicas fisioterapêuticas que visam um trabalho muscular global, o Iso Stretching é uma técnica mais abrangente, que busca o fortalecimento dos diferentes grupos musculares que sustentam o corpo, desenvolvendo a consciência de posições corretas da coluna e, também, da capacidade respiratória ${ }^{6}$.

Já a pompage é uma técnica osteopática desenvolvida por Cathie no intuito de preparar a fascia para então realizar a correção de lesões. Podem ser realizadas com objetivos circulatórios, musculares, articulares e ação calmante ${ }^{7}$. Este recurso fisioterapêutico manual é baseado na respiração e a sua fase expiratória pode contribuir para relaxar além do diafragma, alguns músculos posturais e acessórios da respiração.

Vários estudos têm demonstrado que o uso isolado do Iso Stretching e da pompage atuam na melhora da musculatura respiratória ${ }^{6,8}$, entretanto, ainda são poucos os que investigaram a relação da terapia manual como forma de reabilitação nos indivíduos com DPOC.

Baseando-se nessas premissas, o objetivo do presente trabalho foi verificar os desfechos do uso associado das técnicas de Iso Stretching e pompage sobre a capacidade funcional, força muscular respiratória e a mobilidade torácica de indivíduos portadores de DPOC.

\section{Metodologia}

Saúde (Santa Maria), v.39, n.2, p.161-170, 2013. Desfechos das técnicas de Iso Stretching e Pompage em pacientes com doença pulmonar

Esta pesquisa caracterizou-se como um estudo piloto, realizada na Clínica Escola de Fisioterapia da Universidade Regional do Noroeste do Estado do Rio Grande do Sul - UNIJUÍ. 
Participaram do estudo indivíduos independente do gênero e idade inferior a 75 anos com diagnóstico de DPOC $\left(\mathrm{VEF}_{1} / \mathrm{CVF}<70 \text { e } \mathrm{VEF}_{1} \geq 80\right)^{9}$, encaminhados ao Programa de Reabilitação Pulmonar (PRP) da instituição.

O projeto foi aprovado pelo Comitê de Ética em pesquisa da UNIJUÍ quanto aos seus aspectos metodológicos e éticos, sob parecer $n^{0}$ 070/2010. Após a autorização, a pesquisa ocorreu somente com a assinatura do termo de consentimento livre e esclarecido pelos participantes.

Participaram da pesquisa 10 indivíduos, onde foram constituídos dois grupos: grupo controle (GC) e grupo tratado (GT). Porém, dos 10 indivíduos convidados a participar do estudo, cinco foram excluídos, dois por agudização da doença e três por não comparecerem ao tratamento. Para tanto, a amostra foi randomizada para constituição dos grupos, compondo o GC com $n=3$ e o GT com $n=2$.

Os critérios de inclusão adotados foram: diagnóstico clínico e espirométrico de DPOC segundo os critérios de GOLD ${ }^{9}$, idade inferior a 75 anos, índice de massa corpórea (IMC) >21 $\mathrm{Kg} / \mathrm{m}^{210}$, clinicamente estável nos últimos três meses (sem exacerbação da doença), indivíduos com doenças ortopédicas e reumáticas, que foram submetidos a cirurgias recentes ou que apresentassem alterações cognitivas que impedissem a realização dos testes e os participantes que faltaram por três dias consecutivos ou três dias alternados.

\section{Protocolo de avaliação}

Primeiramente todos os indivíduos realizaram uma anamnese, a fim de verificar as condições gerais de saúde. Posteriormente, foram submetidos às avaliações da capacidade funcional, força muscular respiratória, espirométrica e cirtometria torácica.

Para a avaliação da capacidade funcional, todos os participantes realizaram o teste de caminhada de seis minutos (TC6), teste do degrau (TD) e teste de sentar e levantar (TSL), na ordem descrita. O TC6 foi aplicado de acordo com o protocolo proposto pela ATS Guidelines for the Six-Minute Walk Test ${ }^{11}$. O TD foi utilizado para estimar a tolerância ao exercício, sendo realizado em um degrau único com $20 \mathrm{~cm}$ de altura. $O$ indivíduo foi orientado a subir e descer do degrau por seis minutos, sendo que as recomendações para execução do teste foram as mesmas preconizadas para o TC6. Ao final foi registrado o número de subidas realizadas, sendo considerado "degrau subido" quando o indivíduo posicionava os dois pés apoiados no degrau ${ }^{12,13}$. No TSL foi utilizada uma cadeira com altura padronizada de $46 \mathrm{~cm}$ sem apoio para os braços. 0 paciente realizou o teste com as mãos cruzadas sobre 0 a região do tórax e foi orientado a sentar e levantar da cadeira o mais rápido possível em um período préestabelecido de um minuto, sendo registrado o número de repetições realizadas ${ }^{14}$. As variáveis fisiológicas (frequência cardíaca, saturação periférica de oxigênio, frequência respiratória e pressão arterial) foram coletadas antes e após a aplicação dos testes.

A força muscular respiratória foi mensurada através da pressão inspiratória máxima $\left(\mathrm{Pl}_{\text {máx }}\right)$ e a pressão expiratória máxima $\left(\mathrm{PE}_{\text {máx }}\right)$, realizadas com aparelho manovacuômetro da marca GERAR ${ }^{\circledR}$, graduado de 0 à $150 \mathrm{cmH} 2 \mathrm{O}$ para a PEmáx e de 0 à $-150 \mathrm{cmH}_{2} \mathrm{O}$ para a PImáx, com o indivíduo sentado confortavelmente, fazendo uso de clipe nasal, e antes de se realizar a avaliação foram fornecidas todas as orientações sobre o teste.

Para a mensuração da $\mathrm{PI}_{\text {máx }}$ e $P E_{\text {máx }}$ todos os participantes realizaram pelo menos três manobras reprodutíveis, cada uma mantida por pelo menos um segundo, até que três esforços tecnicamente adequados fossem realizados e com um minuto de intervalo entre

Rev. Saúde (Santa Maria), Santa Maria, v.39, n.2, p.161-170 Jul./Dez.2013. Pasqualoto A.S., et at. 
cada repetição. Para o registro das pressões foi considerado o de valor mais alto, com uma variação de menos de $10 \%$ entre elas. Os valores obtidos por cada indivíduo foram comparados aos seus respectivos valores previstos para a população brasileira ${ }^{15}$.

A espirometria foi realizada com um espirômetro, modelo Micro Medical Limited ${ }^{\circledR}$, de acordo com as diretrizes para realização do teste de função pulmonar ${ }^{16}$. Foram realizados três testes, com variação inferior a 5\%, e o maior valor obtido foi comparado com valores de referência ${ }^{17}$. Os seguintes parâmetros foram obtidos: capacidade vital forçada (CVF) e 0 volume expiratório forçado no primeiro segundo $\left(\mathrm{VEF}_{1}\right)$.

Para a medida da cirtometria torácica o indivíduo permaneceu em posição ortostática e foi instruído a realizar uma expiração máxima para a primeira medida e uma inspiração máxima para a segunda medida, tomando como ponto de referência a linha axilar, mamilar e xifoidiana. A diferença entre a inspiração e a expiração fornece o grau de expansibilidade e de retração dos movimentos toracoabdominais ${ }^{18}$.

Para o monitoramento do grau de dispnéia durante os testes de capacidade funcional foi utilizada a escala analógica de Borg modificada ${ }^{19}$, a qual foi mensurada a cada minuto. É um método muito simples de avaliação e de fácil reprodução em que o indivíduo avaliado indica um valor, através de uma escala visual graduada de 0 a 10, sendo que 0 (zero) representa nenhum grau de dispneia, 3 (três) grau "moderado", 5 (cinco) grau "grave" e 10 (dez) grau "máximo" de dispneia.

\section{Protocolo de intervenção no GT}

O protocolo foi composto de cinco posturas do método Iso Stretching, realizadas na posição deitada e sentada, com utilização de bolas e bastões para diferentes graus de dificuldade e evolução, seguida de nove manobras de pompage.

Inicialmente os participantes foram submetidos às manobras de descolamento do diafragma e estiramento diafragmático, onde cada uma foi mantida por sete ciclos respiratórios profundos, sendo realizado o estímulo manual a cada expiração, garantindo um tempo mínimo de trinta segundos para cada uma ${ }^{20}$, conforme proposto por Pegoraro ${ }^{21}$.

Posteriormente, foram aplicadas as seguintes posturas de Iso Stretching:

- Indivíduo em sedestação, pés paralelos a largura do quadril, tronco devidamente inclinado para frente e coluna ereta com os membros superiores abduzidos e estendidos, punhos em extensão e dedos apontados para cima. 0 indivíduo foi orientado a realizar uma contração isométrica da musculatura abdominal, quadríceps e músculos suspensores da cintura escapular (autocrescimento) seguido de uma expiração, para reger o tempo da manutenção da postura.

- Indivíduo em sedestação, pés paralelos a largura do quadril, joelhos flexionados, tronco inclinado para frente e coluna ereta, mãos apoiadas nos joelhos e punhos em extensão e dedos apontados para cima. 0 indivíduo foi orientado a realizar uma contração isométrica da musculatura abdominal, quadríceps e músculos suspensores da cintura escapular (autocrescimento).

- Indivíduo sentado, pés paralelos a largura do quadril, joelhos flexionados, pelve em anteversão, membros superiores segurando atrás da coluna um bastão horizontalmente.

- Indivíduo sentado, pelve em anteversão, membros superiores sustentando um bastão a

Saúde (Santa Maria), v.39, n.2, p.161-170, 2013. Desfechos das técnicas de Iso Stretching e Pompage em pacientes com doença pulmonar frente do corpo, membros inferiores em abdução flexionados e cruzados, erguer a cabeça ao longo do bastão e "soprar" para o teto, indivíduo fará uma contração isométrica muscular 
abdominal, mantendo o autocrescimento e realizará uma expiração para reger o tempo da manutenção da postura.

- Indivíduo sentado, pelve em anteversão, membros superiores sustentando um bastão a frente do corpo, membros inferiores em abdução, flexionados e cruzados. 0 indivíduo foi orientado a realizar uma contração isométrica muscular abdominal, inclinação anterior do tronco mantendo o autocrescimento e realizará uma expiração para reger 0 tempo da manutenção da postura.

As manobras de pompage foram realizadas, conforme proposto por Bienfait ${ }^{7}$, nas seguintes musculaturas: peitoral menor, peitoral maior, costal inferior, costal superior, escaleno posterior, trapézio superior, elevador da escápula e esternocleidomastóide.

\section{Intervencão no GC}

Nos indivíduos do GC foi aplicado o protocolo de reabilitação pulmonar, comumente usado na instituição, composto por alongamentos globais, aquecimento e treinamento aeróbico em esteira, estabelecendo a intensidade do treinamento pela frequência cardíaca máxima $\left(\mathrm{FC}_{\text {máx }}\right)\left(70 \%\right.$ a $80 \%$ da $\left.\mathrm{FC}_{\text {máx }}\right)$ com incremento semanal na intensidade do exercício, a partir da $\mathrm{FC}_{\text {máx }}$ e da escala Borg modificada, fortalecimento com recursos de theraband, alteres, caneleiras, bastões, associados com exercícios respiratórios, sempre observando os sinais vitais, escala de Borg modificada e saturação periférica de oxigênio.

Ambos os protocolos de intervenção (GT e GC) ocorreram durante quatro semanas, com frequência de duas sessões semanais e com duração média de cinquenta minutos cada. Após o término das intervenções foi realizada a reavaliação, sendo aplicados os mesmos testes descritos.

\section{Análise estatística}

A análise estatística foi realizada no software SPSS 16.0. Os resultados foram expressos em média e desvio padrão. A fim de verificar a normalidade dos dados, o teste Shapiro-Wilk foi aplicado. Para comparar as médias pré e pós intervenção nos grupos, o teste t de Student foi utilizado. Um $p \leq 0,05$ foi adotado como nível de significância.

\section{Resultados}

Participaram do estudo cinco indivíduos com DPOC de grau leve e moderado de acordo com GOLD9. Nas tabelas 1 e 2 estão demonstrados os dados antropométricos e espirométricos basais do GT e GC. Considerando a classificação do IMC pelos parâmetros preconizados pelas diretrizes ${ }^{10}$, destaca-se que os dois grupos apresentaram sobrepeso e com risco aumentado para doença cardiovascular considerando os valores da relação cintura/quadril. 
Tabela 1 - Características antropométricas dos grupos.

\begin{tabular}{ccc}
\hline Variáveis & $\mathrm{GT}(\mathrm{n}=3)$ & $\mathrm{GC}(\mathrm{n}=2)$ \\
\hline Sexo (M/F) & $2 / 1$ & $2 / 0$ \\
Idade (anos) & $70 \pm 6,55$ & $70 \pm 7,07$ \\
Peso (Kg) & $69 \pm 19,1$ & $72,62 \pm 15,37$ \\
Altura (m) & $1,59 \pm 0,15$ & $1,63 \pm 0,15$ \\
IMC (Kg/m2) & $26,68 \pm 2,70$ & $27 \pm 0,94$ \\
$\mathrm{RC} / \mathrm{Q}$ & $1,01 \pm 0,09$ & $1,03 \pm 0,15$ \\
\hline
\end{tabular}

GT: grupo tratado; GC: grupo controle; M: masculino; F: feminino; IMC: índice de massa corporal; RC/Q: relação cintura/quadril.

Tabela 2 - Características espirométricas.

\begin{tabular}{ccc}
\hline Dados espirométricos & GT $(n=3)$ & GC $(n=2)$ \\
\hline CVF(L) & $2,32 \pm 0,51$ & $1,98 \pm 0,87$ \\
pCVF (\%predito) & $72,66 \pm 17$ & $66,5 \pm 3,53$ \\
pVEF1 (\%) & $69,33 \pm 13,42$ & $89 \pm 2,82$ \\
VEF1/CVF & $25,5 \pm 4,93$ & $38,44 \pm 17,38$ \\
\hline
\end{tabular}

GT: grupo tratado; GC: grupo controle; CVF: capacidade vital forçada; pCVF: percentual capacidade vital forçada; pVEF1: percentual do volume expiratório forçado no primeiro segundo pós broncodilatador; VEF1/CVF: relação VEF1/CVF.

$\mathrm{Na}$ análise da força muscular respiratória pré intervenção, realizada pela manovacuometria, foram observados que ambos os grupos não apresentavam fraqueza muscular respiratória de acordo com os valores previstos ${ }^{15}$, porém no GT foram encontrados valores ligeiramente menores para 0 previsto da força muscular inspiratória e expiratória quando comparados com o GC. Os resultados da PImáx após a intervenção evidenciou incremento tanto nos valores absolutos como nos percentuais previstos de força no GT em dois indivíduos ( $G T p=0,21$ e $G C p=0,7$ ). Comportamento diferente foi observado no $G C$ onde apenas um indivíduo apresentou melhora na $\mathrm{PE}_{\text {máx }}$. Para a $\mathrm{PI}_{\text {máx }}$ foi observado um acréscimo nos valores para todos os indivíduos do grupo GT após 4 semanas de intervenção (GT $p=0,28$ e GC p $=0,8$ ) (Tabela 3).

Tabela 3 - Força muscular respiratória pré intervenção.

\begin{tabular}{ccc}
\hline Testes & GT $(\mathrm{n}=3)$ & $\mathrm{GC}(\mathrm{n}=2)$ \\
\hline PImáx(cm H2O) & $75 \pm 7,55$ & $108 \pm 25,45$ \\
\%PImáx & $82,61 \pm 26,23$ & $108,59 \pm 10,48$ \\
PEmáx (cm H2O) & $72,66 \pm 12,66$ & $113,5 \pm 3,53$ \\
\%PEmáx & $77,17 \pm 15,28$ & $104,56 \pm 2,25$ \\
\hline
\end{tabular}

Saúde (Santa Maria), v.39, n.2, p.161-170, 2013. Desfechos das técnicas de Iso Stretching e Pompage em pacientes com doença pulmonar 
Com relação aos testes de capacidade funcional, os pacientes do GT apresentaram um aumento na distância percorrida no TC6 $(p=0,004)$ e um melhor desempenho no TD6 $(p=0,027)$ após a intervenção. Enquanto que os pacientes do grupo controle diminuíram a distancia percorrida no TC6 $(p=0,029)$ e no TD6 ( $p=0,014)$, no TSL não apresentaram alterações significativas. (Tabela 4)

Quando comparados os dois grupos (GT e GC) foi encontrada diferença significativa pós intervenção para as variáveis TC6 $(p=0,004)$ e TSL $(p=0,027)$ no $G T$.

Tabela 4 - Testes de capacidade funcional antes e após o protocolo de intervenção.

\begin{tabular}{ccccc}
\hline Variável & GT $(n=3)$ & Teste T & GC (n=2) & Teste T \\
& Media \pm DP & & Media \pm DP & \\
\hline TC6 \\
(avaliação) & $352,0 \pm 119,0$ & NS & $479,50 \pm 53,03$ & NS \\
TC6 $_{\text {(reavaliação) }}$ & $425,33 \pm 47,38^{*}$ & 0,004 & $\# 430,50 \pm 27,52$ & 0,029 \\
TD6 $_{\text {(avaliação) }}$ & $80,33 \pm 48,33$ & NS & $108,0 \pm 60,8$ & 0,014 \\
TD6 $_{\text {(reavaliação) }}$ & $91,00 \pm 42,67$ & NS & $82,50 \pm 74,24$ & NS \\
TSL $_{\text {(avaliação) }}$ & $17,67 \pm 5,5$ & NS & $23,5 \pm 0,7$ & NS \\
\hline
\end{tabular}

*Teste T para GT; \#teste T para GC; NS = não significativo; $p<0,05$ existe diferença estatisticamente significativa entre as médias pós intervenção.

Ao analisar os resultados da expansibilidade torácica foi verificado que independente do nível avaliado (axilar, mamilar e xifoidiano) foi encontrado um aumento na maioria dos indivíduos do GT, porém sem significância estatística $(p=0,2)$

\section{Discussão}

Neste estudo foi possível observar que o uso associado das técnicas de Iso Stretching e pompage preconizam, como um dos seus principais objetivos, o trabalho respiratório. Com 0 protocolo de tratamento proposto, constatou-se um aumento na expansibilidade torácica, na força muscular e na capacidade funcional dos indivíduos portadores de DPOC.

0 resultado em comum das duas técnicas vem da idéia de que a estimulação diafragmática promove um melhor desempenho mecânico do músculo e, consequentemente, uma ventilação pulmonar mais satisfatória e com menor esforço muscular ${ }^{22}$. A intervenção fisioterapêutica, visando à correção postural e a reeducação diafragmática, promovem restabelecimento da função pulmonar pela melhora da mecânica ventilatória e do trabalho muscular diafragmático com redução do trabalho respiratório ${ }^{23}$.

Os exercícios do método Iso Stretching e as manobras de pompage promoveram a melhora na função dos músculos respiratórios na amostra estudada. Corroborando com resultados encontrados, 0 estudo de Brandt et al. ${ }^{24}$ avaliou o impacto da aplicação do Iso Stretching sobre o comportamento respiratório toracoabdominal numa população sadia. A técnica foi aplicada em seis jovens sedentários, previamente submetidos a uma avaliação respiratória pontuada e a análise angular fotogramétrica da inspiração e expiração máximas Rev. Saúde (Santa Maria), Santa Maria, v.39, n.2, p.161-170 Jul./Dez.2013. na postura ortostática e caracterização do padrão muscular ventilatório utilizado. As posturas de Iso Stretching foram aplicadas em sessões diárias de 45 minutos, durante 10 dias. Os ${ }_{\text {Iss }}^{2236-5843}$ 
resultados encontrados foram compatíveis com a melhora da atuação diafragmática durante a mobilização de médios e altos volumes respiratórios e sugerem que o método pode promover impacto efetivo e mensurável sobre a função respiratória de seus praticantes.

Engel et al. ${ }^{25}$ descreveu que 0 uso da terapia manual em indivíduos normais e fumantes foi capaz de melhorar a função pulmonar, além de minimizar alguns efeitos respiratórios negativos ao exercício. Neste estudo as posturas do Iso Stretching e as manobras fasciais proporcionaram efeitos benéficos sobre os músculos respiratórios, avaliado pela manovacuometria, evidenciando aumento nos valores desta variável.

Quanto à força muscular inspiratória, estudos ${ }^{26}$ observaram a influência da fraqueza muscular generalizada e da desvantagem mecânica dos músculos inspiratórios sobre a obtenção da $\mathrm{PI}_{\text {máx }}$ em pacientes com DPOC. Através do protocolo adotado neste estudo, o GT obteve aumento importante da força muscular respiratória, tanto da PImáx como da $\mathrm{PE}_{\text {máx }}$, após a intervenção, sugerindo melhora na vantagem mecânica, indo de acordo com a lei tensão-comprimento ${ }^{27}$.

Os pacientes submetidos ao programa de Iso Stretching e pompage durante quatro semanas também apresentaram aumento da capacidade ao exercício submáximo. Diferentes estudos mostraram que pacientes com DPOC submetidos a um programa de treinamento físico aeróbio apresentaram melhora na capacidade de exercício após um período de treinamento de dois mese ${ }^{28}$. É interessante notar que neste estudo apenas um participante teve diminuição da distância percorrida no TC6. Embora inesperada, a melhora no TC6 pode ser devida à alteração da capacidade ventilatória avaliada, pelo aumento da mobilidade da caixa torácica ou por uma melhora do padrão respiratório.

Embora o TD6 e o TSL tenham seu uso restrito, alguns programas de reabilitação têm adotado eles para avaliar a capacidade funcional nos portadores de DPOC29. Esses testes foram adotados na presente pesquisa para contribuir na avaliação da capacidade funcional dos indivíduos, sendo que os resultados evidenciaram pouca modificação nos valores pré e pós intervenção, diferentemente ao analisar a capacidade funcional pelo TC6.

Em estudo recente, foi constatado que o Iso Stretching aplicado em uma população de indivíduos com diagnóstico de patologias variadas, evidenciou o ganho na expansibilidade torácica após 10 sessões da técnica ${ }^{30}$. Ao analisar as respostas na expansibilidade pelo uso de técnicas manuais, em um protocolo de oito sessões, os resultados obtidos foram semelhantes ao estudo descrito por Yokohama ${ }^{31}$.

\section{Conclusão}

Através deste estudo foi possível observar que após as quatro semanas de intervenção houve um aumento na força muscular respiratória e na expansibilidade torácica, mais evidente no GT. Ainda, em relação à capacidade funcional, o GT também apresentou melhora, representado pelo aumento da distância percorrida após a intervenção no TC6.

Limitações do estudo: 0 tamanho da amostra não foi calculado, pelo qual não foi possível dizer que os resultados encontrados possam ser extrapolados ao demais portadores

Saúde (Santa Maria), v.39, n.2, p.161-170, 2013. Desfechos das técnicas de Iso Stretching e Pompage em pacientes com doença pulmonar de DPOC. Em função da exclusão de alguns participantes, no GT permaneceram somente os pacientes com obstrução moderada ao fluxo aéreo e no GC pacientes com obstrução leve, 0 
que pode ter interferido nos resultados. Além disso, é importante ressaltar, que estudos futuros avaliem outras formas de intervenção, buscando contribuir no manejo deste grupo de pacientes.

\section{Referências Bibliográficas}

1. Sclauser Pessoa IMB, Parreira VF, Lorenzo VAP, Reis MAS, Costa D. Análise da hiperinsuflação pulmonar dinâmica $(\mathrm{HD})$ após atividade de vida diária em pacientes com doença pulmonar obstrutiva crônica. Rev Bras Fisioter. 2007;11(6):469-74.

2. Sobradillo V, Zenarruzabetia E, Cabriada V. Atualização em pneumologia: DPOC. Barcelona: Permanyer Publications; 1998.

3. Souchard E. O stretching global ativo: a reeducação postural global a serviço do esporte. São Paulo: Manole; 1996.

4. Mador MJ, Bozkanat E. Skeletal muscle dysfunction in chronic obstructive pulmonary disease. Respir Res. 2001;2:216-24.

5. Pasqualoto AS, Knorst MM. Manifestações sistêmicas e causas de intolerância ao exercício na DPOC. In: Moraes EB, Franz LB, organizadores. Coleção Saúde Coletiva - Doenças e agravos não transmissíveis. ljuí: UNIJUI; 2011.

6. Batista J, Lima M. O Iso-Stretching no ganho de expansibilidade torácica e força muscular respiratória em indivíduos saudáveis. In: II Seminário de Fisioterapia: Iniciação Cientííca; 2008 maio 5-6; Foz do Iguaçu : Faculdade Uniamérica; 2008.

7. Bienfait M. Bases elementares técnicas de terapia manual e osteopatia. $3^{a}$ ed. São Paulo: Summus; 1997.

8. Achour Júnior A. Exercícios de alongamento: anatomia e fisiologia. Barueri: Manole; 2000.

9. Global Initiative for Chronic Obstrutive Lung Diseas (GOLD) [Internet]. 2013. Disponível em: http://www.goldcopd.org.

10. Schols AM, Soeters PB, Dingemans AM, Mostert R, Frantzen PJ, Wouters EF. Prevalence and characteristics of nutritional depletion in patients with stable COPD eligible for pulmonary rehabilitation. Am Rev Respir Dis. 1993;147:1151-6.

11. ATS Committee on Proficiency Standards for Clinical Pulmonary Function Laboratories. ATS statement: guidelines for six-minute walk test. Am J Respir Crit Care Med. 2002;166:111-7.

12. Dal Corso S, Duarte SR, Neder JA, Malaguti C, Fuccio MB, Castro Pereira CA, Nery LE. A step test to assess exercise-related oxygen desaturation in interstitial lung disease. Eur Respir J. 2007;29(2):330-6.

13. Pasqualoto AS. Comparação das respostas fisiológicas no teste de exercício cardiopulmonar e em três testes de exercício submáximo em pacientes com doença pulmonar obstrutiva crônica [tese]. Porto Alegre: Universidade Federal do Rio Grande do Sul; 2009.

14. Ozalevli S, Ozden A, Itil O, Akkoclub O. Comparison of the Sit-to-Stand Test with 6 min walk test in patients with chronic obstructive pulmonary disease. Respir Med. 2007;101(2):286-93.

15. Neder JA, Andreoni S, Lerario MC, Nery LE. Reference values for lung function tests. II. Maximal respiratory pressures and voluntary ventilation. Braz J Med Biol Res. 1999;32(6):719-27.

16. Sociedade Brasileira de Pneumologia e Tisiologia. Diretrizes para teste de função pulmonar. J Pneumol. 2002;28(Supl 3):S1-S238.

Rev. Saúde (Santa Maria), Santa Maria, v.39, n.2, p.161-170 Jul./Dez.2013. Pasqualoto, A. S., et al.

17. Pereira CAC, Barreto SP, Simões JG, Pereira FWL, Gerstler JG, Nakatani J. Valores de referência para espirometria em uma amostra da população brasileira adulta. J Pneumol. 1992;18(1):10-22. 
18. Chiavegato LD, Jardim JR, Faresin SM, Juliano Y. Alterações funcionais respiratórias na coleciscectomia por via laparoscópica. J Pneumol. 2000;26(2):69-76.

19. Borg GA. Psychophysical bases of perceived exertion. Med Sci Sports Exerc. 1982;14(5):377-81.

20. Bandy WD, Irion JM. The effect of time on static stretch on the flexibility of the hamstring muscles. Phys Ther. 1994;74(9):845-50.

21. Pegoraro M. Influência da aplicação de técnicas fisioterapêuticas manuais direcionadas à cadeia muscular respiratória sobre o limiar anaeróbico em atletas de natação [monografia]. Cascavel: Unioeste, Curso de Fisioterapia; 2004.

22. Costa D. Fisioterapia respiratória básica. São Paulo: Atheneu; 1999.

23. Ribeiro EC, Soares LM. Avaliação espirométrica de crianças portadoras de respiração bucal antes e após intervenção fisioterapêutica. Fisioter Bras. 2003;4(3):163-7.

24. Brandt AC, Ricieri DV, Griesbach LES. Repercussões respiratórias da aplicação da técnica de isostretching em indivíduos sadios. Fisioter Bras. 2004;5(2):103-10.

25. Engel RM, Vemulpad S. Progression to chronic obstructive pulmonary disease (COPD): could it be prevented by manual therapy and exercise during the 'at risk' stage (stage 0)? Med Hypotheses. 2009;72(3):288-90.

26. Wen AS, Woo MS, Keens TG. How many maneuvers are required to measure maximal inspiratory pressure accurately?. Chest. 1997;111(3):802-7.

27. De Troyer A, Estenne M. Functional anatomy of the respiratory muscles. Clin Chest Med. 1988;9(2):17593.

28. Goldstein RS, Gort EH, Stubbing D, Avendano MA, Guyatt GH. Randomized controlled trial of respiratory rehabilitation. Lancet. 1994;344(8934):1394-7.

29. Casaburi R, Porszasz J, Burns MR, Carithers ER, Chang RS, Cooper CB. Physiologic benefits of exercise training in rehabilitation of patients with severe chronic obstructive pulmonary disease. Am J Respir Crit Care Med. 1997;155(5):1541-51.

30. Vilaró J, Resqueti VR, Fregonezi GAF. Avaliação clínica da capacidade do exercício em pacientes com doença pulmonar obstrutiva crônica. Rev Bras Fisioter. 2008;12(4):249-59.

31. Yokohama TV. A prática do Iso-stretching na melhora da expansibilidade toracopulmonar, verificada através da espirometria e da cirtometria [monografia]. Cascavel: Unoeste, Curso de Fisioterapia; 2004.

\section{Adriane Schmidt Pasqualoto}

Endereço para correspondência - Av. Roraima, nº 1000. Bairro Camobi, CEP 97105-900. Santa Maria, RS, Brasil.

E-mail: aspasqualoto@hotmail.com

Currículo Lattes: http://lattes.cnpq.br/3243310050281330

Enviado em 01 de maio de 2013.

Publicado em 12 de novembro de 2013.

Saúde (Santa Maria), v.39, n.2, p.161-170, 2013. 